\title{
Memoria de trabajo y habilidades de parendizaje verbal en estudiantes de cuarto grado de primaria
}

\section{Working memory and verbal learning skills in fourth grade elementary students}

\author{
Claudia Patricia Rivera Sánchez ${ }^{1}$
}

Lizeth Ramos Acosta ${ }^{2}$

Chrystian Mauricio Pérez Serna ${ }^{3}$

Olga Lizbeth Salamanca ${ }^{4}$

Unidad Central del Valle del Cauca

\begin{abstract}
$1 \quad$ Licenciada en Educación Básica con Énfasis en Lenguas Extranjeras. Magíster en Neuropsicología y Educación. Doctoranda en Educación. Docente Tiempo Completo y Coordinadora de la Práctica Docente de la Licenciatura en Lenguas Extranjeras con Énfasis en Inglés de la Unidad Central del Valle del Cauca, Uceva.

CORREO: cprivera@uceva.edu.co ORCID: https://orcid. org/0000-0003-1867-2852

GOOGLE SCHOLAR: https://scholar.google.es/citations?user=VAy280OAAAAJ\&amp:hl=es
\end{abstract}

$2 \quad$ Licenciada en Lenguas Modernas, Especialista en Docencia para la Educación Superior y Magíster en Educación Superior. Docente Tiempo Completo y Coordinadora de la Licenciatura en Lenguas Extranjeras con Énfasis en Inglés de la Unidad Central del Valle del Cauca, Uceva. CORREO: Iramos@uceva.edu.co ORCID: https://orcid. org/0000-0002-9035-1992.GOOGLE SCHOLAR: https:// scholar.google.com.co/citations? user $=y 25 d 6 b c A A A A-$ J\&hl=es

3 Licenciado en Educación Básica con Énfasis en Lenguas Extranjeras. Maestrante en Bilingüismo y Educación. Docente Hora Cátedra de la Unidad Central del Valle del Cauca. Coordinador Académico del Colegio Bilingüe Hispanoamericano de Tuluá. CORREO: cperez@uceva.edu.co ORCID: https://orcid.org/0000-0002-1282-6526

$4 \quad$ Licenciada en Educación Básica con Énfasis en Lenguas Extranjeras. Maestrante en Bilingüismo y Educación. Docente Tiempo Completo de la Unidad Central del Valle del Cauca. Correo: osalamanca@uceva.edu.co ORCID: https://orcid.org/0000-0002-2261-9870

\section{RESUMEN}

El presente proyecto busca Analizar la relación de la memoria de trabajo con las habilidades de aprendizaje verbal en estudiantes de cuarto grado de primaria de la institución educativa Moderna del municipio de Tuluá, utilizando para ello herramientas como el Trail Making Test, el Taveci y el Promedio académico. Se midieron en total 10 variables cuantitativas, de las cuales se obtuvieron 24 relaciones significativas para los estudiantes de alto desempeño y 6 relaciones significativas para los estudiantes que presentan bajo desempeño, y una variable que se analizó gráficamente que fue el índice de aprendizaje.

Para lograr una producción y comprensión del lenguaje, el cerebro realiza una serie de procesos que activan zonas específicas de la memoria, ya que debe acudir al almacenamiento 
de conceptos ya sean fonológicos, morfológicos, semánticos, sintácticos o pragmáticos para lograr una relación eficaz entre lo que se habla y la intención con la cual se efectúa la comunicación. Por lo tanto, la memoria es un factor fundamental para la comprensión del lenguaje oral y escrito (Baddeley, 1999).

En este proceso la memoria de trabajo juega un papel importante, dado que hace referencia a la retención y manipulación de información durante cortos períodos de tiempo, en los cuales se realizan acciones más complejas como el razonamiento y la resolución de problemas, además de ser la encargada de manejar conductas según estadios emocionales del organismo; así mismo, permite realizar operaciones de codificación y elaboración de la información para que se integre debidamente a lo que ya posee el ser humano, en ella se evocan los recuerdos que guarda la memoria a largo plazo, lo que le permite al sujeto realizar tareas determinadas (Tirapu \& Munóz Céspedes, 2005).

En este orden de ideas, un proceso que hace parte importante en los procesos de memoria a corto y largo plazo, incluyendo la memoria semántica y la discriminación auditiva, es el aprendizaje verbal, pues se refiere a procesos cognitivos que relacionan la memoria y el conocimiento semántico, ya que se ejercita la memoria semántica a corto y largo plazo para la recuperación de información. Aquí juegan un papel importante la conciencia fonológica, el reconocimiento sonido palabra y la comprensión del lenguaje oral, pues son factores implicados en el aprendizaje verbal.

PALABRAS CLAVE: Memoria de trabajo, rendimiento académico, aprendizaje verbal, funciones ejecutivas.

\section{ABSTRACT}

This project seeks to analyze the relationship of working memory with verbal learning skills in fourth grade primary school students of the Moderna educational institution in the municipality of Tuluá, using tools such as the Trail Making Test, the Taveci and the Academic Average. A total of 10 quantitative variables were measured, of which 24 significant relationships were obtained for high-performance students and 6 significant relationships for students with low-performance, and a variable that was graphically analyzed was the learning index.

In order to achieve the production and understanding of language, the brain performs a series of processes that activate specific areas of memory, since it must go to the storage of concepts whether phonological, morphological, semantic, syntactic or pragmatic to achieve an effective relationship between what is spoken and the intention with which The communication is carried out. Therefore, memory is a vital factor for understanding both oral and written language (Baddeley A. , 1999).

In this process, working memory plays an important role, as it refers to the retention and manipulation of information for short periods of time, in which more complex actions such as reasoning and problem solving are carried out, in addition to being in charge of managing behaviors according to emotional stages of the organism, it also makes it possible to carry out operations of coding and processing of information so that it is properly integrated with what is already possessed by the human being, recalling the memories that the long-term memory keeps, allowing the subject to perform specific tasks (Tirapu \& Muñoz, 2005).

Along this line, the process that plays a crucial roll in the short and long-term memory processes including semantic memory and auditory discrimination is verbal learning, seeing that this one refers to cognitive processes that relatememory and semantic knowledge, since the semantic memory is exercised in the short and long term for the recovery of information. 5 
Here they play an important role, phonological awareness, word sound recognition and oral language comprehension, as they are factors involved in verbal learning.

KEYWORDS: Working memory, academic performance, verbal learning, executive functions.

\section{INTRODUCCIÓN}

Para lograr una producción y comprensión del lenguaje, el cerebro realiza una serie de procesos que activan zonas específicas de la memoria, ya que debe acudir al almacenamiento de conceptos ya sean fonológicos, morfológicos, semánticos, sintácticos o pragmáticos para lograr una relación eficaz entre lo que se habla y la intención con la cual se efectúa la comunicación. Por lo tanto la memoria es un factor fundamental para la comprensión del lenguaje tanto oral como escrito (Baddeley, 1999).

En este proceso, la memoria de trabajo juega un papel importante, ya que, hace referencia a la retención y manipulación de información durante cortos períodos de tiempo, en los cuales se realizan acciones más complejas como lo son el razonamiento y la resolución de problemas, además de ser la encargada de manejar conductas según estadios emocionales del organismo, así mismo permite realizar operaciones de codificación y elaboración de la información para que se integre debidamente a lo que ya posee el ser humano, en ella se evocan los recuerdos que guarda la memoria a largo plazo, lo que le permite al sujeto realizar tareas determinadas (Tirapu \& Munóz Céspedes, 2005).

En este orden de ideas, un proceso que hace parte importante en los procesos de memoria a corto y largo plazo, incluyendo la memoria semántica y la discriminación auditiva es el aprendizaje verbal, pues se refiere a procesos cognitivos que relacionan la memoria y el conocimiento semántico, ya que se ejercita la memoria semántica a corto y largo plazo para la recuperación de información. Aquí juegan un papel importante, la conciencia fonológica, el reconocimiento sonido palabra y la comprensión del lenguaje oral, pues son factores implicados en el aprendizaje verbal.

Teniendo en cuenta lo anterior, la presente investigación busca Analizar la relación de la memoria de trabajo con las habilidades de aprendizaje verbal en estudiantes de cuarto grado de primaria que presentan alto y bajo rendimiento académico de la institución educativa Moderna del municipio de Tuluá, utilizando para ello herramientas como el Trail Making Test, el Taveci y el Promedio académico. Se midieron en total 10 variables cuantitativas, de las cuales se obtuvieron 6 relaciones significativas para los estudiantes de alto rendimiento y 24 relaciones significativas para los estudiantes que presentan bajo rendimiento, y una variable que se analizó gráficamente que fue el índice de aprendizaje.

Esta investigación se enfoca en el estudio de variables que hacen parte de los diferentes procesos de pensamiento de los seres humanos, lo que permite un conocimiento más amplio de las funciones de aprendizaje, lo cual le permitirá a la comunidad educativa tener una aproximación más pertinente para el desarrollo de las diferentes competencias que plantean los planes del Ministerio de Educación, los cuales tiene como objetivo el mejoramiento de los procesos de pensamiento de los educandos, para que ellos puedan desarrollar diferentes competencias para la vida.

\section{MARCO TEÓRICO Y CONCEPTUAL}

Debido a que la memoria de trabajo pertenecer a las Funciones Ejecutivas; las cuales se relacionan con el aprendizaje verbal, se hace necesario presentar fundamentación teórica para definirlas. 


\section{FUNCIONES EJECUTIVAS}

Las funciones ejecutivas, según Luria (1980) son las encargadas de controlar el comportamiento de las personas, pues el cerebro establece metas para cada actividad. Por lo tanto, el autor agrupó el funcionamiento del cerebro en una serie de unidades que cumplen diferentes funciones, las cuales permite llevar a cabo diferentes tareas.

Según Baddeley, (1986) las funciones ejecutivas son un director y programador de procesos cognitivos como la autorregulación, la anticipación, planificación, conducta, autocontrol y retroalimentación.

Según Reynolds y Horton (2008), las funciones ejecutivas son la capacidad de generar repuestas a los estímulos externos.

Según Pineda (2015), El desarrollo más grande de las funciones ejecutivas ocurre entre los 6 y los 8 años de edad, durante este periodo los niños adquieren la capacidad de autorregular sus comportamientos y conductas, así mismo, empiezan a desarrollar la capacidad de fijarse metas y anticipar eventos sin necesitar instrucciones externas. Así mismo se encuentra que a la edad de 12 años los niños ya poseen una organización cognoscitiva muy similar o cercana a la que se puede observar en los adultos, aunque el desarrollo completo de la función se presenta a los 16 años.

\section{CLASIFICACIÓN DE LAS FUNCIONES EJECUTIVAS}

Según Baddeley y Hitch (1974) las funciones ejecutivas son las encargadas de regular conductas en dominios cognitivos, planeación, organización, perseveración, inhibición, fluidez e iniciación.

Por otro lado, Según Portellano (2005), las Funciones ejecutivas son las encargadas de la inteligencia, la atención, la memoria, el lenguaje, la flexibilidad mental, el control motor y la conducta., estos procesos son regulados por los lóbulos frontales.

$\checkmark$ Inteligencia: La inteligencia está regulada por el lóbulo prefrontal y lóbulos frontales, y hace referencia a la capacidad del ser humano para entender, razonar, tomar decisiones, planificar acciones, crear ideas y opiniones.

$\checkmark \quad$ La atención: Las capacidades del control voluntario de la atención están ubicadas en la corteza prefrontal, y hace referencia a la aplicación de los sentidos a una tarea determinada.

$\checkmark$ Memoria de trabajo: Se ubica en los lóbulos frontales, específicamente en el área prefrontal y se refiere a la capacidad de almacenar información temporal para manipularla, transformarla, etc.

$\checkmark \quad$ Lenguaje: También llamado fluidez verbal o aprendizaje verbal, el cual está ubicado en los lóbulos frontales, allí se encuentra el área de Broca, la cual permite una articulación y decodificación del lenguaje. Además, están implicados el lóbulo occipitotemporal, donde está ubicada el área de Wernicke, que es donde se le da significado al lenguaje y su comprensión fonológica. También están implicados los lóbulos occipitales, ya que allí se decodifica el lenguaje por medio de la vía visual, la cual ayuda al reconocimiento de palabras y de factores extralingüísticos que están implicados en la comprensión pragmática del lenguaje. Por otro lado, se encuentran implicadas las vías de lectura léxica y subléxica, las cuales decodifican el lenguaje escrito.

$\checkmark$ Flexibilidad mental: Están implicada el área dorsolateral de los lóbulos frontales. $Y$ hace referencia a la capacidad de acomodación o adaptación que tiene el ser humano ante el cambio de estímulos, innovando o inhibiendo funciones. 
$\checkmark$ Control motor: Hace referencia a la programación y ejecución de frecuencias motoras, y aquí están implicados los lóbulos frontales y parietales.

$\checkmark$ Regulación o control emocional: Esta es una de las funciones más importantes de la condición humana en su campo personal, la cual hace referencia al control emocional como respuesta a estímulos. Aquí están implicados los lóbulos frontales, el área prefrontal y lóbulos temporales, así como el sistema límbico.

\section{BASES NEUROANATÓMICAS DE LAS FUNCIONES EJECUTIVAS}

Las funciones ejecutivas implican en su mayoría los lóbulos frontales y corteza prefrontal, ya que son los encargados del control de impulsos, el juicio, la producción del lenguaje, la memoria de trabajo, las funciones motoras, la conducta, la planificación y coordinación (Portellano, 2005). También están implicados los lóbulos parietal, encargado de la información sensorial, táctil, la manipulación de objetos, y la relación con los otros lóbulos para la información del lenguaje y el conocimiento numérico; el lóbulo occipital, que es donde reside la corteza visual, recibe y procesa información visual y le da coherencia al entorno en relación con los otros lóbulos; y el lóbulo temporal, que es el encargado de procesar información auditiva, y olfativa, controla el equilibrio, la coordinación y la memoria, relacionándola con estímulos sonoros.

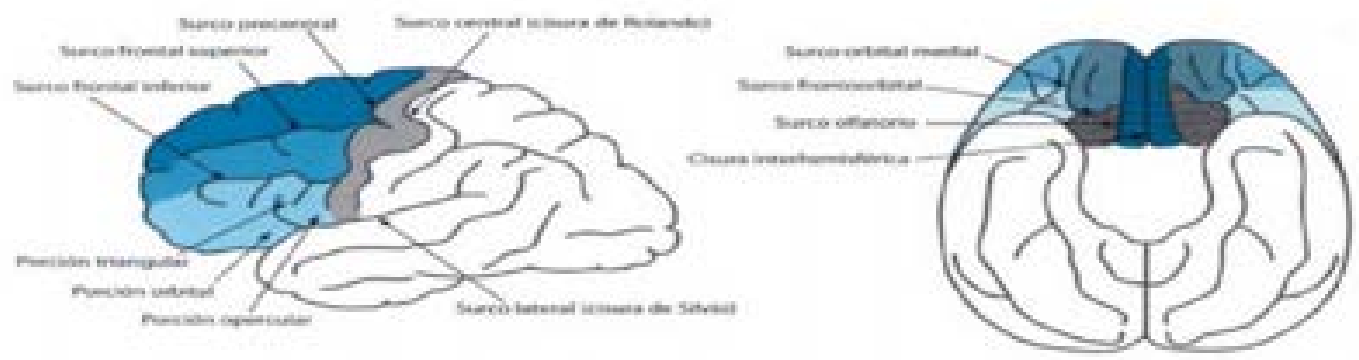

Bases neuroanatómicas de las Funciones Ejecutivas (Minaño, 2014).

\section{MEMORIA DE TRABAJO}

La memoria de trabajo hace referencia a la retención y manipulación de información durante cortos períodos de tiempo, en los cuales se realizan acciones más complejas como lo son el razonamiento y la resolución de problemas, además de ser la encargada de manejar conductas según estadios emocionales del organismo (Tirapu \& Munóz Céspedes, 2005).

A lo largo de los años, el concepto de memoria ha evolucionado, ya no se concibe como un único almacén, sino que se ha subdividido en varios almacenes para el procesamiento de cada tipo de información, en relación con esto, aparece la memoria a corto plazo y dentro de esta, la memoria de trabajo, que hace referencia no solo al almacenamientos de información en corto períodos de tiempo, sino además a la manipulación de esta para tareas determinadas (Baddeley A. , Working memory: Theories, models and controversies., 2012)

El modelo de memoria de trabajo o memoria operativa, planteada por Baddeley y Hitch (1974) contemplaba tres subversiones de la memoria de trabajo: Ejecutivo Central, Bucle fonológico o lazo articulatorio y la agenda visoespacial. Posteriormente, en el año 2000, Baddeley añadió una nueva subdivisión, el bufer episódico. De esta forma, la Memoria de trabajo estaría compuesta por cuatro subdivisiones. 


\section{SUBDIVISIÓN DE LA MEMORIA DE TRABAJO}

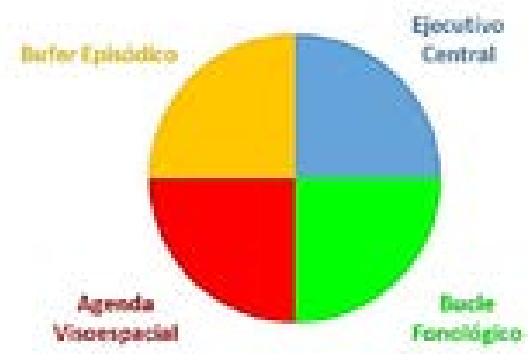

\section{EJECUTIVO CENTRAL}

Según Baddeley (1996) el ejecutivo central asigna la atención a las tareas que realiza el ser humano, este tiene cuatro funciones que son: 1. Coordinar tareas independientes, almacenamiento y procesamiento de la información. 2. Recuperar información de la memoria a largo plazo. 3. Selección de información específica. 4. Cambio de tarea y estrategias para la coordinación de operaciones.

Subdivisión de la memoria de trabajo.

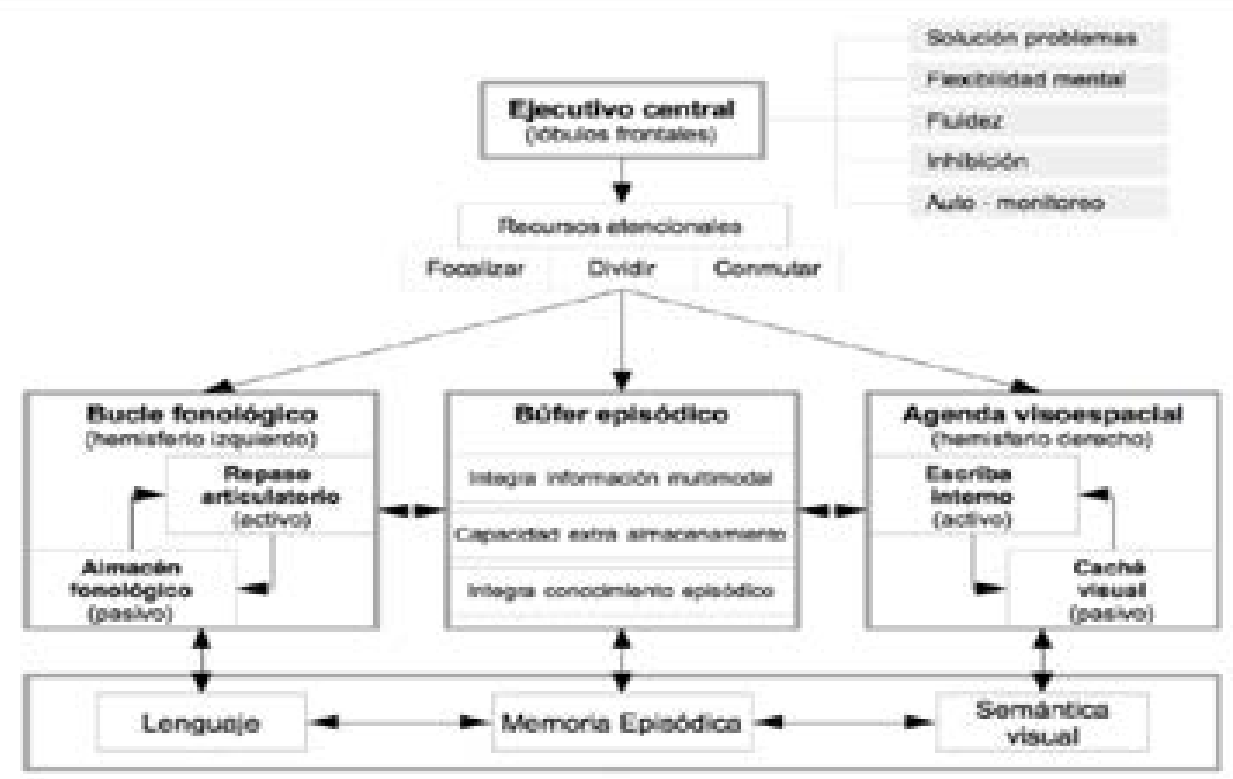

Modelo Multicomponente de memoria de trabajo de Baddeley (Sardinero Peña, 2015).

\section{BUCLE FONOLÓGICO O LAZO} ARTICULATORIO

Es el componente encargado de retener información verbal en forma acústica de manera temporal. Este está compuesto por 1. Un almacén de información acústica, la cual tiene una permanencia de 3 segundos, a menos que tenga un refuerzo de repetición. 2 . Un almacén de información acústico-verbal, (habla) que por medio de la articulación repetitiva almacena la información de forma indefinida.

\section{AGENDA VISOESPACIAL}

Este componente es el encargado de retener información visual, por medio de las imágenes, es similar al bucle fonológico, solo que este maneja información visual en vez de Sonora. Baddeley sostiene que el cerebro tiene la capacidad de procesar información por separado; por un lado la visual y por otro la especial, lo cual permite la relación de los movimientos visuals-cinestésicos (Baddeley A. , 2003).

\section{BUFER EPISÓDICO}

Este es el encargado de manejar información 
multimodal, no solo se enfoca en imágenes o sonidos, sino que puede combinar información del medio. Otra de sus características es que permite el intercambio de información entre la memoria a largo plazo y la de trabajo.

\section{BASES NEUROPSICOLÓGICAS DE LA MEMORIA DE TRABAJO.}

Bases neuroanatómicas de la memoria de trabajo.

\begin{tabular}{|c|l|}
\hline COMPONENTE & \multicolumn{1}{|c|}{ LOCALIZACIÓN } \\
\hline Bucle articulatorio & $\begin{array}{l}\text { Mantenimiento: Corteza prefrontal ventrolateral } \\
\text { izquierda: anterior (información semántica) y } \\
\text { posterior (información fonológica) } \\
\text { Almacenamiento: Áreas corticales posteriores } \\
\text { izquierdas para el habla (Broca) y el lenguaje } \\
\text { (Werniccke). }\end{array}$ \\
\hline Agenda Visoespacial & $\begin{array}{l}\text { Mantenimiento: Corteza prefrontal ventrolateral } \\
\text { derecha. } \\
\text { Almacenamiento: Áreas parieto-opcipitales } \\
\text { derechas para la visión. }\end{array}$ \\
\hline Ejecutivo Central & $\begin{array}{l}\text { Corteza prefontal dorsolateral } \\
\end{array}$ \\
\hline
\end{tabular}

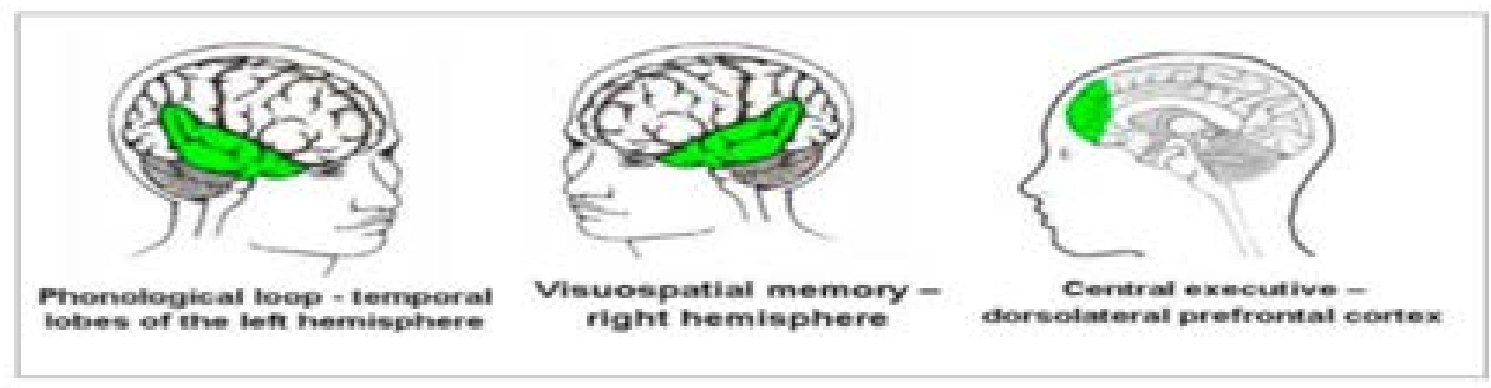

Bases neuropsicológicas de la memoria de trabajo (Martínez, 2014).

\section{APRENDIZAJE VERBAL}

La producción de palabras requiere un conjunto de funciones cognitivas, en la cual la memoria juega un papel importante, ya que, se hace un proceso de recuperación de la información léxica y semántica para producir un discurso, frases o simplemente secuencias de palabras. Para esto es necesario un organizado proceso cognitivo, atención focal, sostenida y procesos de planificación (Lezak, Howieson, Loring, Hannay, \& Fischer, 2004).
Por lo tanto, el aprendizaje verbal hace referencia a procesos cognitivos que relacionan la memoria y el conocimiento semántico, ya que se ejercita la memoria semántica a corto y largo plazo para la recuperación de información. Aquí juegan un papel importante, la conciencia fonológica, la reconocimiento sonido palabra y la comprensión del lenguaje oral, pues son factores implicados en el aprendizaje verbal.

$\checkmark \quad$ Concienciafonológica: Es lacapacidad de analizar y segmentar componentes del habla 
(palabras, rimas, sílabas, fonemas, sonidos) para realizar algún tipo de operación sobre estos. Esta permite la manipulación grafemafonema, y es crucial para el aprendizaje de la lectura y de una segunda lengua. (Morrais , Alegria, \& Content, 1987).

$\checkmark$ Reconocimiento sonido-palabra: Es la capacidad que tiene el ser humano para relacionar sonidos con grafemas o símbolos que forman un concepto, de esta forma se hace un proceso de recuperación al escuchar un sonido y recordar un concepto, o de almacenamiento cuando se establece una relación de sonido-concepto para permitirle al cerebro guardar esa información.

$\checkmark$ Comprensión del lenguaje oral: Aquí están implicados procesos más complejos, debido a que ya no solo se relacionan sonidos y conceptos, sino que se tienen en cuenta las reglas gramaticales y sintácticas, secuencia de palabras y categorías gramaticales para darle sentido al discurso; además se hace una relación de los signos lingüísticos y paralingüísticos (tono de voz, gesticulación, postura) para darle una comprensión global al mensaje que se está comunicando; aquí tienen lugar el discurso semántico y pragmático.

\section{BASES NEUROANATÓMICAS DEL} APRENDIZAJE VERBAL

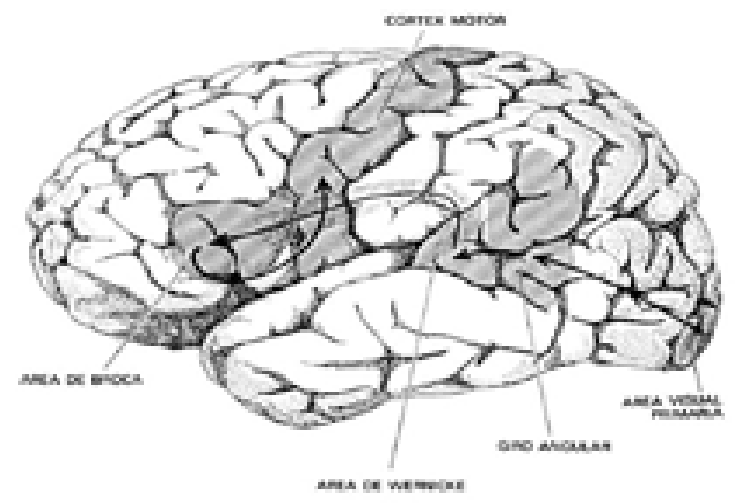

Modelo neurolingüística de Wernicke-

Geschwind (Llisterri, 2018).
Norman Gesch wind (1926-1984) propone las regiones corticales que se encargarían de los procesos del lenguaje, establece así, una ruta para el procesamiento del lenguaje oral (pronunciar una palabra oída) y para el escrito (pronunciar una palabra leída). Además de los centros receptivos visuales y auditivos y áreas motoras articulatorias:

$\checkmark \quad$ Área de Wernicke para la comprensión del lenguaje.

$\checkmark$ Circunvolución angular, donde se integran impulsos visuales y auditivos y permite elaborar la representación sonora de la palabra leída.

$\checkmark$ Fascículo arqueado que comunica las áreas de Wernicke y de Broca.

$\checkmark$ Área de Broca para la articulación del lenguaje.

Corteza motora primaria que produce los movimientos fonoarticulatorios. 
Población y Muestra

La distribución de la población se representa de la siguiente manera:

Distribución de la muestra Alto desempeño académico.

\begin{tabular}{|l|c|c|c|c|c|c|c|c|c|c|}
\hline Género & Frecu. & Porcen. & & $\begin{array}{c}\text { Nro. } \\
\text { Estud }\end{array}$ & Med. & Median. & Moda & Desv. Est & V. Mín. & V. Max \\
\hline Mascul. & 4 & $40 \%$ & Edad & 4 & 9,75 & 10 & 10 & 0,5 & 9 & 10 \\
\hline Femen. & 6 & $60 \%$ & Género & 6 & 9,333 & 9 & 9 & 0,516398 & 9 & 10 \\
\hline
\end{tabular}

Distribución de la muestra Bajo desempeño.

\begin{tabular}{|c|c|c|c|c|c|c|c|c|c|c|}
\hline Género & Frecu. & Porcen. & & Nro. & Med. & Median. & Moda & Desv.Est & V. Mín. & $\begin{array}{c}\text { V. } \\
\text { Max }\end{array}$ \\
\hline Mascul. & 4 & $40 \%$ & Edad & 4 & 9,5 & 9,5 & 9 & 0,57735 & 9 & 10 \\
\hline Femen. & 6 & $60 \%$ & Género & 6 & 9,5 & 9,5 & 9 & 0,547723 & 9 & 10 \\
\hline
\end{tabular}

\section{Instrumentos}

Memoria de Trabajo: Trail Making Test (TMT) Parte B

Aprendizaje Verbal: Test Taveci

Desempeño académico: Promedio de resultados académicos.

\section{ANÁLISIS DE RESULTADOS}

En el siguiente análisis se abordará el proceso que se llevó a cabo, con relación a cada uno de los objetivos, para lo cual se utilizó el complemente de Excel, EZAnalize 3.0.

\section{MEMORIA DE TRABAJO EN ESTUDIANTES CON ALTO RENDIMIENTO ACADÉMICO.}

El primer objetivo hace referencia a identificar el nivel de memoria de trabajo en la muestra, lo cual se logró por medio de la aplicación del TMT (Trail Making Test) parte B.

Análisis descriptivos de MT: Memoria de trabajo.

\begin{tabular}{ccccccc}
\hline \multicolumn{2}{c}{ MT } & & \\
\hline Nro. Estu. & Media & Mediana & Moda & Desviación estándar & Valor Mínimo & $\begin{array}{c}\text { Valor } \\
\text { Máximo }\end{array}$ \\
10 & 90,500 & 83,000 & 0 & 21,701 & 65,000 & 129,000 \\
\hline
\end{tabular}

La tabla anterior muestra el análisis de la variable Memoria de Trabajo en estudiantes que presentan alto rendimiento académico, teniendo en cuenta que el promedio debe ser 70 segundos para tener un buen nivel de Memoria de Trabajo, y como puntaje deficiente está el resultado mayor a 273 segundos, se puede concluir los siguientes:

El nivel mínimo se encuentra de 65 segundos, un nivel que supera el promedio que presenta la prueba, sin embargo, se encuentra que el 
valor de la moda no es posible observarlo ya que, ninguno de los datos correspondiente al promedio de tiempo de la prueba se repite, se encuentra que la media equivale a 90,500 lo que los sitúa más cerca del promedio del buen nivel, aunque presentan falencias que deben mejorar, pues se puede observar que el valor máximo que obtuvieron los estudiantes fue de 129 segundos, un puntaje muy cerca del nivel deficiente, que requiere de intervención neuropsicológica para mejorar sus procesos de memoria de trabajo. Finalmente, se puede observar una desviación estándar de 21,701 lo cual indica que los resultados tienen un nivel medio de dispersión.

MEMORIA DE TRABAJO EN ESTUDIANTES CON BAJO RENDIMIENTO ACADÉMICO.

Análisis descriptivos de MT: Memoria de trabajo.

\begin{tabular}{ccccccc}
\hline & \multicolumn{6}{c}{ MT } \\
\hline Nro. Estu. & Media & Mediana & Moda & $\begin{array}{c}\text { Desviación } \\
\text { estándar }\end{array}$ & Valor Mínimo & Valor Máximo \\
10 & 92,600 & 89,500 & 0 & 18,069 & 60,000 & 124,000 \\
\hline
\end{tabular}

La tabla anterior muestra el análisis de la variable Memoria de Trabajo en estudiantes que presentan bajo rendimiento académico, teniendo en cuenta que como se mencionó anteriormente, el promedio debe ser 70 segundos para tener un buen nivel de Memoria de Trabajo, y como puntaje deficiente está el resultado mayor a 273 segundos, se puede concluir los siguientes:

El nivel mínimo se encuentra de 60 segundos, un nivel excelente que supera el promedio que presenta la prueba, sin embargo, se encuentra, que el valor de la moda no es posible observarlo debido a que, ninguno de los datos correspondiente al promedio de tiempo de la prueba se repite, se encuentra que la media equivale a 90,500 lo que los sitúa más cerca del promedio del buen nivel, aunque presentan falencias que deben mejorar, pues se puede observar que el valor máximo que obtuvieron los estudiantes fue de 124 segundos, un puntaje muy cerca del nivel deficiente, que requiere de intervención neuropsicológica para mejorar sus procesos de memoria de trabajo. Finalmente, se puede observar una desviación estándar de 18,069 lo cual indica que los resultados tienen un nivel medio de dispersión.

\section{APRENDIZAJE VERBAL EN ESTUDIANTES CON ALTO RENDIMIENTO ACADÉMICO.}

El Segundo objetivo hace referencia a identificar el nivel de aprendizaje verbal de la población objeto de estudio. Para esto, se aplicó el test TAVECI el cual mide las variables: 1) Recuerdo libre inmediato. 2) Recuerdo libre a corto plazo. 3) Recuerdo a corto plazo con claves semánticas. 4) Recuerdo libre a largo plazo. 5) Recuerdo a largo plazo con claves semánticas. 6) Reconocimiento a largo plazo. 7) Recencia y Primacía. 8) Discriminación auditiva. A continuación se presentará un análisis descriptivo de cada una de ellas. 
Análisis descriptivo del Aprendizaje Verbal Alto rendimiento académico.

APRENDIZAJE VERBAL

\begin{tabular}{|l|c|c|c|c|c|c|c|}
\hline & $\begin{array}{c}\text { Nro. } \\
\text { Estudi. }\end{array}$ & Media & Mediana & Moda & $\begin{array}{c}\text { Desviación } \\
\text { Estándar }\end{array}$ & $\begin{array}{c}\text { Valor } \\
\text { Mínimo }\end{array}$ & $\begin{array}{c}\text { Valor } \\
\text { Máximo }\end{array}$ \\
\hline RLI & 10 & 9,330 & 9,600 & 9,600 & 1,357 & 6,300 & 11,000 \\
\hline RL-CP & 10 & 10,300 & 10,000 & 10,000 & 1,829 & 8,000 & 14,000 \\
\hline RCL-CP & 10 & 9,200 & 9,500 & 12,000 & 3,048 & 3,000 & 13,000 \\
\hline RL-LP & 10 & 10,000 & 10,000 & 9,000 & 1,563 & 8,000 & 12,000 \\
\hline RCL-LP & 10 & 9,800 & 9,000 & 9,000 & 2,251 & 7,000 & 14,000 \\
\hline PRIMACíA & 10 & 32,800 & 33,500 & 33,000 & 6,250 & 19,000 & 41,000 \\
\hline RECENCIA & 10 & 27,300 & 27,500 & 28,000 & 4,270 & 22,000 & 35,000 \\
\hline $\begin{array}{l}\text { DISC. } \\
\text { AUDITIVA }\end{array}$ & 10 & 5,320 & 4,400 & 4,400 & 2,822 & 0,0 & 11,100 \\
\hline
\end{tabular}

RLI: Recuerdo libre inmediato RL-CP: Recuerdo libre a corto plazo RCL-CP: Recuerdo libre a corto plazo con claves semánticas. RL-LP: Recuerdo libre a largo plazo. RCL-LP: Recuerdo libre a largo plazo con claves semánticas. DISC AUDITIVA: Discriminación auditiva.

APRENDIZAJE VERBAL EN ESTUDIANTES CON bajo RENDIMIENTO ACADÉMICO.

Análisis descriptivo del Aprendizaje Verbal Bajo rendimiento académico

APRENDIZAJE VERBAL

\begin{tabular}{|l|c|c|c|c|c|c|c|}
\hline & $\begin{array}{c}\text { Nro. } \\
\text { Estudi. }\end{array}$ & Media & Mediana & Moda & $\begin{array}{c}\text { Desviación } \\
\text { Estándar }\end{array}$ & $\begin{array}{c}\text { Valor } \\
\text { Mínimo }\end{array}$ & $\begin{array}{c}\text { Valor } \\
\text { Máximo }\end{array}$ \\
\hline RLI & 10 & 7,790 & 7,700 & 0 & 2,410 & 4,000 & 11,600 \\
\hline RL-CP & 10 & 8,800 & 9,000 & 12,000 & 2,781 & 5,000 & 12,000 \\
\hline RCL-CP & 10 & 8,700 & 9,000 & 10,000 & 1,767 & 5,000 & 11,000 \\
\hline RL-LP & 10 & 8,400 & 7,500 & 13,000 & 3,373 & 4,000 & 13,000 \\
\hline RCL-LP & 10 & 9,300 & 9,500 & 8,000 & 1,889 & 6,000 & 12,000 \\
\hline PRIMACÍA & 10 & 29,300 & 27,000 & 19,000 & 8,206 & 19,000 & 42,000 \\
\hline RECENCIA & 10 & 22,000 & 25,500 & 29,000 & 10,708 & 2,000 & 36,000 \\
\hline $\begin{array}{l}\text { DISC. } \\
\text { AUDITIVA }\end{array}$ & 10 & 10,880 & 12,200 & 17,800 & 6,929 & 2,200 & 17,800 \\
\hline
\end{tabular}

RLI: Recuerdo libre inmediato RL-CP: Recuerdo libre a corto plazo RCL-CP: Recuerdo libre a corto plazo con claves semánticas. RL-LP: Recuerdo libre a largo plazo. RCL-LP: Recuerdo libre a largo plazo con claves semánticas. DISC AUDITIVA: Discriminación auditiva. 
ANÁLISIS CORRELACIONAL - estudiantes con alto desempeño académico.

En la siguiente tabla se puede observar el análisis correlacional de las variables.

Análisis correlacional de las variables - Alto desempeño académico.

\begin{tabular}{|c|c|c|c|c|c|c|c|c|c|}
\hline & MT & RLI & RL-CP & RCL-CP & RL-LP & RCL-LP & PRIMACÍA & RECENCIA & DISC.A \\
\hline MT & 1 & & & & & & & & \\
\hline$N$ & - & & & & & & & & \\
\hline$P$ & - & & & & & & & & \\
\hline RLI &,- 139 & 1 & & & & & & & \\
\hline$N$ & 10 & - & & & & & & & \\
\hline$P$ & ,70 & - & & & & & & & \\
\hline RL-CP & 242 & ,775* & 1 & & & & & & \\
\hline$N$ & 10 & 10 & - & & & & & & \\
\hline$P$ &, 50 & 01 & - & & & & & & \\
\hline RCL-CP & 141 & ,450 & ,447 & 1 & & & & & \\
\hline$N$ & 10 & 10 & 10 & - & & & & & \\
\hline$P$ & ,70 & 19 & ,20 & - & & & & & \\
\hline RL-LP & 170 & ,451 & ,505 & ,676* & 1 & & & & \\
\hline$N$ & 10 & 10 & 10 & 10 & - & & & & \\
\hline$P$ & ,64 & 19 & 14 & ,03 & - & & & & \\
\hline RCL-LP &,- 105 & ,348 & 205 & ,719* & ,410 & 1 & & & \\
\hline$N$ & 10 & 10 & 10 & 10 & 10 & - & & & \\
\hline$P$ & ,77 & ,32 & ,57 & ,02 & ,24 & - & & & \\
\hline PRIMACÍA & 118 & ,892* & ,677* & ,516 & 341 & 305 & 1 & & \\
\hline$N$ & 10 & 10 & 10 & 10 & 10 & 10 & - & & \\
\hline$P$ & ,75 & ,00 & ,03 & 13 & ,33 & 39 & - & & \\
\hline RECENCIA &,- 451 & ,733* & 613 & 174 & 383 & ,215 & , 381 & 1 & \\
\hline$N$ & 10 & 10 & 10 & 10 & 10 & 10 & 10 & - & \\
\hline$P$ & 19 & ,02 & ,06 & ,63 & ,27 &, 55 & ,28 & - & \\
\hline DISC.A & ,343 &,- 080 &,- 195 &,- 113 &,- 564 &,- 165 & 295 &,- 553 & 1 \\
\hline$N$ & 10 & 10 & 10 & 10 & 10 & 10 & 10 & 10 & - \\
\hline$P$ & ,33 & ,83 & ,59 & ,76 & ,09 & ,65 & ,41 & 10, & - \\
\hline
\end{tabular}

MT: Memoria de trabajo. RLI: Recuerdo libre inmediato. RL-CP: Recuerdo libre a corto plazo. RCL-CP: Recuerdo a corto plazo con claves semánticas. RL-LP: Recuerdo libre a largo plazo. RCLLP: Recuerdo a largo plazo con claves semánticas. DISC. A: Discriminación auditiva.

Las relaciones significativas están resaltadas con un asterisco $\left({ }^{*}\right)$. 
La tabla anterior muestra un total de cinco (5) correlaciones, las cuales evidencian una relación fuerte y directa.

\section{ÍNDICE DE APRENDIZAJE - ALTO DESEMPEÑO ACADÉMICO}

\section{índice de aprendizaje - Alto desempeño académico.}

En la figura anterior se puede observar el índice de aprendizaje de los estudiantes que presentan alto desempeño académico, el cual tiene un comportamiento ascendente desde el ensayo 1 hasta el ensayo 5 , evidenciando un mejoramiento en la capacidad de recuerdo libre inmediato. Sin embargo, siguiendo el transcurso del índice, se puede notar un descenso desde el recuerdo a corto plazo hasta el recuerdo a largo plazo, lo que significa que hubo más dificultad en recordar las palabras después de que se presentó la lista $B$, la cual obtenía palabras completamente diferentes a las de la lista A. Aquí, la mayor dificultad se encuentra en el recuerdo a largo plazo, pues tiene una puntuación de 10.0, por debajo del ensayo 6 referente al recuerdo a corto plazo, esto puede deberse a que este índice se mide justo después de transcurridos los 20 minutos en los cuales los estudiantes trabajaron en una actividad no verbal.

De lo anterior se puede concluir que, existen falencias en la recuperación de la información, pues aún en su mayor puntuación, existe un déficit de 5 palabras que no se lograron recuperar.

ANÁLISIS CORRELACIONAL - estudiantes con bajo desempeño académico.

En la siguiente tabla se puede observar el análisis correlacional de las variables.

Análisis correlacional de las variables - Bajo desempeño académico.

\begin{tabular}{|c|c|c|c|c|c|c|c|c|c|c|}
\hline & & MT & RLI & RL-CP & RCL-CP & $\begin{array}{l}\text { RL- } \\
\text { LP }\end{array}$ & $\begin{array}{l}\text { RCL- } \\
\text { LP }\end{array}$ & PRIMACÍA & RECENCIA & DISC.A \\
\hline \multirow[t]{3}{*}{ MT } & & 1 & & & & & & & & \\
\hline & $N$ & - & & & & & & & & \\
\hline & $P$ & - & & & & & & & & \\
\hline \multirow[t]{3}{*}{ RLI } & &,- 455 & 1 & & & & & & & \\
\hline & $N$ & 10 & - & & & & & & & \\
\hline & $P$ & ,19 & - & & & & & & & \\
\hline \multirow[t]{3}{*}{ RL-CP } & &,- 555 & ,819* & 1 & & & & & & \\
\hline & $N$ & 10 & 10 & - & & & & & & \\
\hline & $P$ & ,10 & ,00 & - & & & & & & \\
\hline \multirow[t]{3}{*}{ RCL-CP } & &,- 317 &, $905^{*}$ & ,823* & 1 & & & & & \\
\hline & $N$ & 10 & 10 & 10 & - & & & & & \\
\hline & $P$ & ,37 & ,00 & , 00 & - & & & & & \\
\hline \multirow[t]{3}{*}{ RL-LP } & &,- 364 & ,949* &, $827^{*}$ & ,824* & 1 & & & & \\
\hline & $N$ & 10 & 10 & 10 & 10 & - & & & & \\
\hline & $P$ & ,30 & ,00 & , 00 & ,00 & - & & & & \\
\hline \multirow[t]{3}{*}{ RCL-LP } & &,- 481 & ,872* & ,795* & ,796* & ,851* & 1 & & & \\
\hline & $N$ & 10 & 10 & 10 & 10 & 10 & - & & & \\
\hline & $P$ & ,16 & ,00 & ,01 & ,01 & ,00 & - & & & \\
\hline PRIMACÍ & &,- 602 & ,759* & ,874* & ,712* &, $734^{*}$ &, $725^{\star}$ & 1 & & \\
\hline & $N$ & 10 & 10 & 10 & 10 & 10 & 10 & - & & \\
\hline
\end{tabular}




\begin{tabular}{|c|c|c|c|c|c|c|c|c|c|}
\hline$P$ & ,07 & ,01 & , 00 & ,02 & ,02 & ,02 & - & & \\
\hline RECENCIA &,- 253 & ,891* & ,537 & ,769* & ,806* & ,725* & ,397 & 1 & \\
\hline$N$ & 10 & 10 & 10 & 10 & 10 & 10 & 10 & - & \\
\hline$P$ & , 48 & , 00 & ,11 & ,01 & , 00 & ,02 & ,26 & - & \\
\hline DISC.A & ,524 &,$- 735^{*}$ &,$- 709^{*}$ &,$- 673^{*}$ &,$- 705^{*}$ &,$- 675^{*}$ &,- 603 &,- 627 & 1 \\
\hline$N$ & 10 & 10 & 10 & 10 & 10 & 10 & 10 & 10 & - \\
\hline$P$ & ,12 & ,02 & ,02 & ,03 & ,02 & ,03 & ,06 & ,05 & - \\
\hline
\end{tabular}

MT: Memoria de trabajo. RLI: Recuerdo libre inmediato. RL-CP: Recuerdo libre a corto plazo. $R$ CLCP: Recuerdo a corto plazo con claves semánticas. RL-LP: Recuerdo libre a largo plazo. RCL-LP: Recuerdo a largo plazo con claves semánticas. DISC. A: Discriminación auditiva.

Las relaciones significativas están resaltadas con un asterisco $\left({ }^{*}\right)$.

La tabla anterior evidencia un total de veinticuatro (24) correlaciones, de las cuales diecinueve son fuertes y directas y cinco (5) son fuertes en indirectas.

\section{ÍNDICE DE APRENDIZAJE - BAJO DESEMPEÑO ACADÉMICO}

\section{Índice de aprendizaje - Alto desempeño académico.}

En la figura anterior se puede observar el índice de aprendizaje de los estudiantes que presentan bajo desempeño académico, el cual tiene un comportamiento ascendente desde el ensayo 1 hasta el ensayo 5, evidenciando un mejoramiento en la capacidad de recuerdo libre inmediato. Sin embargo, siguiendo el transcurso del índice, se puede notar un descenso desde el recuerdo a corto plazo hasta el recuerdo a largo plazo, lo que significa que hubo más dificultad en recordar las palabras después de que se presentó la lista B, la cual obtenía palabras completamente diferentes a las de la lista A. Aquí, la mayor dificultad se encuentra en el recuerdo a largo plazo, pues tiene una puntuación de 8.4, por debajo del ensayo 6 referente al recuerdo a corto plazo, esto puede deberse a que este índice se mide justo después de transcurridos los 20 minutos en los cuales los estudiantes trabajaron en una actividad no verbal.

De lo anterior se puede concluir que, existen falencias en la recuperación de la información, pues aún en su mayor puntuación, existe un déficit de 5 palabras que no se lograron recuperar.

\section{DISCUSIÓN DE CONSTRUCCIÓN Y CRÍTICA}

La presente investigación tuvo como objetivo Analizar la relación de la memoria de trabajo con las habilidades de aprendizaje verbal en estudiantes de cuarto grado de primaria de una institución educativa pública del municipio de Tuluá.

Teniendo en cuenta que, la memoria de trabajo permite realizar operaciones de codificación y elaboración de la información para que se integre debidamente a lo que ya posee el ser humano, lo que le permite al sujeto realizar tareas determinadas, utilizando conceptos que ha adquirido durante su desarrollo y utilizando aquello que le permite solucionar un problema en un momento determinado permitiéndole al estudiante comprender los textos, resolver los problemas, calcular mentalmente, escribir, manejar otra lengua, etc. $Y$ es aquí donde radica la importancia de la memoria de trabajo en el proceso de aprendizaje (Baddeley A. , 1999).

Ahora, se encuentra el aprendizaje verbal, el cual se encarga directamente del procesamiento de información lingüística, ya que, permite la recuperación de la información léxica y semántica para producir un discurso, frases o simplemente secuencias de palabras. Este 
aprendizaje verbal está influenciado por una serie de factores o procesos cognitivos y emocionales que le permiten al estudiante desempeñar adecuadamente su proceso de aprendizaje. En este aprendizaje juega un papel importante la conciencia fonológica, el reconocimiento sonido palabra y la comprensión del lenguaje oral, pues son factores implicados en el aprendizaje verbal.

Por otro lado se encuentra la investigación de López (2017) la cual concluye que se hace evidente la relación que existe entre memoria de trabajo, en su componente verbal (bucle fonológico) y los procesos implicados en el lenguaje, al fin y al cabo, el lenguaje es aprendizaje y la memoria es la encargada de mantener dicha información en funcionamiento y contribuir a su correcto uso.

Ahora, para realizar el respectivo análisis de cada una de las variables implicadas en el proceso y recolección de datos para esta investigación, es fundamental tener en cuenta que se evaluará principalmente la media y la desviación estándar obtenida ya que la media permitirá identificar el promedio obtenido en cada grupo (Alto desempeño académico y bajo desempeño), y por su parte la desviación estándar permitirá identificar que tan dispersos o sólidos son los resultados obtenidos.

Por lo anterior, se encuentra que la media obtenida en la prueba TMT que mide memoria de trabajo para los estudiantes que presentan alto desempeño académico fue de 90,6 y los estudiantes de bajo desempeño obtuvieron una media de 92,6 en este caso, teniendo en cuenta que para que se obtenga un resultado adecuado dentro de la prueba el estudiante debe tardar máximo 75 segundos para resolver el ejercicio, se puede concluir que los estudiantes que presentan alto desempeño académico obtuvieron mejores resultados que los estudiantes que presentan bajo desempeño, debido a que estos se encuentran más cerca del promedio de 70 a 75 segundos que presenta la prueba para obtener un buen nivel. Sin embargo cuando se analiza la desviación estándar se encuentra que los estudiantes que presentan alto rendimiento académico presentan una desviación estándar más alta, equivalente a 21,791 y los estudiantes que presentan bajo desempeño obtuvieron 18,069 lo que nos muestra que los estudiantes de alto desempeño obtuvieron un resultado mayor a los estudiantes que presentan bajo rendimiento, y esto permite concluir que pesar de que los estudiantes de bajo rendimiento tuvieron una media más alta la desviación estándar muestra que los resultados obtenidos por estos son más sólidos.

Ahora, se encuentra que la media obtenida en la prueba que mide aprendizaje verbal TAVECI para los estudiantes que presentan alto desempeño académico fue de 14,188 y los estudiantes que presentan bajo desempeño obtuvieron 13,146. En este caso teniendo en cuenta que el resultado de cada una de las variables que mide la prueba se realiza de forma ascendente, excepto Discriminación auditiva, la cual se mide por porcentaje de errores, es decir entre más valor, mayor número de errores. Se puede concluir que, los estudiantes que presentan alto rendimiento académico tuvieron mejores resultados en la prueba, ya que su media está más cerca de 15 que es el número total de palabras que el estudiante debe recordar, Así mismo, se encuentra que la desviación estándar de estos estudiantes es de 2,924 y los estudiantes de bajo desempeño obtuvieron 4,758 lo que permite identificar que los resultados obtenidos en esta prueba por los estudiantes que presentan alto desempeño son más sólidos.

Por lo anterior, es necesario hablar de las correlaciones obtenidas en ambas pruebas, para esto es necesario tener en cuenta que la prueba de aprendizaje verbal TAVECI cuenta con 8 variables, estas son: $R$ LI: Recuerdo libre inmediato. RL-CP: Recuerdo libre a corto plazo. 
RCL-CP: Recuerdo a corto plazo con claves semánticas. RL-LP: Recuerdo libre a largo plazo. RCL-LP: Recuerdo a largo plazo con claves semánticas. Y DISC. A: Discriminación auditiva. Ahora, después de analizar cada uno de los resultados obtenidos mediante la aplicación de ambas pruebas en estudiantes que presentan alto desempeño académico y estudiantes que presentan bajo desempeño académico se puede identificar que a pesar de que los resultados obtenidos por los estudiantes de alto desempeño fueron mejores en ambas pruebas que aquellos que presentan bajo desempeño, quienes presentan mayor correlaciones son los estudiantes de bajo desempeño, es decir que los resultados obtenidos por estos son más sólidos, ya que se observa que las variables se afectan mutuamente, mientras que por su parte alto rendimiento tuvo solo 6 correlaciones, por el contrario se encuentra que bajo desempeño cuenta con 24 correlaciones lo que quiere decir que los resultados de las variables de bajo rendimientosonmejores, es decir alto rendimiento académico tienen mejor memoria de trabajo, calificaciones y habilidades de aprendizaje verbal, pero los resultados obtenidos por los estudiantes que presentan bajo rendimiento son más sólidos aunque inferior a los resultados de los estudiantes de alto rendimiento. Esto enfocado hacia la necesidad del docente se encuentra que es más pertinente tener dentro del aula a estudiantes que presenten el mismo nivel cognitivo, ya que así este puede utilizar y diseñar estrategias para todos, pero cuando se presenta en el aula estudiantes que presentan un nivel cognitivo demasiado superior entre unos y otros no es posible utilizar estrategias para el grupo en general, ya que no será posible obtener resultados adecuados.

Así mismo, es necesario tener en cuenta el índice de aprendizaje obtenido por cada uno de los grupos evaluados, en esta se puede observar que los estudiantes de alto desempeño iniciaron recordando 5.1 palabras y terminaron recordando 10, los estudiantes de bajo desempeño por su parte iniciaron recordando 4.4 palabras y terminaron recordando 8.4 palabras lo que muestra que los estudiantes que recordaron más palabras en total son aquellos que presentan alto desempeño ya que estos tuvieron un mayor incremento de recuerdo equivalente a 4.9 palabras.

Por último, se puede concluir que los estudiantes que presentan alto desempeño académico tuvieron mejores resultados que aquellos que presentan bajo desempeño, lo que muestra la relación que existe entre el nivel de memoria de trabajo y las habilidades de aprendizaje verbal con el nivel académico de los estudiantes, entre mejor rendimiento académico presentan los estudiantes mejores resultados se obtienen referentes a la memoria de trabajo y las habilidades de aprendizaje verbal que adquieren y desarrollan los educandos.

\section{REFERENCIAS BIBLIOGRÁFICAS}

Baddeley, A. (1986). Working Memory . Oxford: Clarendon Press.

Baddeley, A. (1996). The fractionation of working memory. Proceeding of the national academic of sciences of the United States of América, 93, 13468-13472.

Baddeley, A. (1999). Memoria Humana: Teoría y práctica. Madrid: Mc Graw-Hill.

Baddeley, A. (2003). Working memor y and lenguaje: An overview. Journal of comunication , 189-208.

Baddeley, A. (2012). Working memory: Theories, models and controversies. Annual review of psychology, 63, 1-29.

Baddeley, A., \& Hitch, G. (1974). Working memory. The psychology of learning and motivation: 8, New York: Academic Press, 47-89. 
Lezak, M., Howieson, D., Loring, D., Hannay, H., \& Fischer, J. (2004). Neuropsychological assessment. New York: NY, US.

Luria, A. (1980). Conciencia y Lenguaje. Madrid: Pablo del Rio.

Morrais , J., Alegria, J., \& Content, A. (1987). The Rela- tionship between Segmental Analysis and Al-phabetic Literacy: An Interactive. Current Psychology of Cognition, 7,, 415-438.

Pineda, D. A. (2 de 12 de 2015). researchgate. net. Obtenido de researchgate. net: $\quad$ https://www.researchgate. net/profile/David_Pineda6/ publication/267939106_LA_FUNCION_ EJECUTIVA_Y_SUS_TRASTORNOS/ links/565f202308ae4988a7be8e4b.pdf

Portellano, J. (2005). Introducción a La Neuropsicología. Madrid: Mc Graw Hill.

Reynolds, , C., \& Horton, A. (2008). Assessing executive functions: A life-span perspective. Psychology in the Schools, 45(9), 875-892.

Tirapu, U., \& Munóz Céspedes, J. (2005). Memoria y funciones ejecutivas. Revista de Neurología, 41,, 475-484. 